\title{
A radio survey of Galactic center clouds
}

\author{
E. A. C. Mills ${ }^{1}$, C. C. Lang ${ }^{2}$, M. R. Morris ${ }^{3}$, J. Ott ${ }^{1}$, N. Butterfield ${ }^{2}$, \\ D. Ludovici ${ }^{2}$, S. Schmitz ${ }^{2}$ and A. Schmiedeke ${ }^{4}$
}

${ }^{1}$ National Radio Astronomy Observatory, USA (email: bmills@aoc.nrao.edu) ${ }^{2}$ Dept. of Physics \& Astronomy, University of Iowa, USA ${ }^{3}$ Dept. of Physics \& Astronomy, University of California-Los Angeles, USA ${ }^{4}$ I. Physikalisches Institut, Universität zu Köln, Germany

\begin{abstract}
We present a radio survey of molecules in a sample of Galactic center molecular clouds, including M0.25+0.01, the clouds near Sgr A, and Sgr B2. The molecules detected are primarily $\mathrm{NH}_{3}$ and $\mathrm{HC}_{3} \mathrm{~N}$; in Sgr B2-N we also detect non-metastable $\mathrm{NH}_{3}$, vibrationally-excited $\mathrm{HC}_{3} \mathrm{~N}$, torsionally-excited $\mathrm{CH}_{3} \mathrm{OH}$, and numerous isotopologues of these species. $36 \mathrm{GHz}$ Class I $\mathrm{CH}_{3} \mathrm{OH}$ masers are ubiquitous in these fields, and in several cases are associated with new $\mathrm{NH}_{3}(3,3)$ maser candidates. We also find that $\mathrm{NH}_{3}$ and $\mathrm{HC}_{3} \mathrm{~N}$ are depleted or absent toward several of the highest dust column density peaks identified in submillimeter observations, which are associated with water masers and are thus likely in the early stages of star formation.
\end{abstract}

Keywords. Galaxy: center — molecular data — radio lines: ISM — techniques: interferometric

The central 300 parsecs of the Galaxy contain one of the largest reservoirs of molecular gas in the Galaxy. Thus far, however, the large-scale distribution and kinematics of this molecular gas have only been probed at arcminute $(\sim 2-3 \mathrm{pc})$ resolutions (e.g., Bally et al. 1987, Jones et al. 2013). This survey is a first step toward a uniform study of Galactic center gas on sub-parsec scales. Ultimately, these survey data will probe the temperatures, densities, and kinematics of a sample of clouds at $2-3^{\prime \prime}(\sim 0.1 \mathrm{pc})$ resolution.

\section{Observations and data calibration}

Observations were made using the new WIDAR correlator in the hybrid DnC array of the Karl G. Jansky Very Large Array (VLA). Ka-band data (27-36 GHz) were observed on 2012 January 7 and 13, and K-band data (24-25 GHz) on 2012 January 8 and 14. The data consist of 18 pointings toward 6 clouds (Sgr B2 M\&N, M0.25+0.01, M-0.11-0.08, $\mathrm{M}-0.02-0.07$, the CND, and $\mathrm{M}-0.13-0.08)$.

The survey was designed to cover a large number of $\mathrm{NH}_{3}$ transitions, which trace gas with densities $\gtrsim 10^{3} \mathrm{~cm}^{-3}$, and can be used to measure kinetic temperature. Eight transitions of $\mathrm{NH}_{3}$ with energies from 20 to $840 \mathrm{~K}$ above the ground state are observed, which are collectively sensitive to a wide range of gas temperatures. In addition, the observations cover multiple transitions of $\mathrm{HC}_{3} \mathrm{~N}$ and $\mathrm{CH}_{3} \mathrm{OH}$.

The observations in each band ( $\mathrm{K}$ and $\mathrm{Ka}$ ) are divided into two separate, continuous subbands $0.86 \mathrm{GHz}$ wide, each comprised of 7 spectral windows. The typical spectral resolution is $250 \mathrm{kHz}\left(\sim 3 \mathrm{~km} \mathrm{~s}^{-1}\right)$, however for three spectral windows, covering (1) the $\mathrm{NH}_{3}(1,1)$ and $(2,2)$ lines and their hyperfine structure, $(2)$ the $36.1 \mathrm{GHz} \mathrm{CH}_{3} \mathrm{OH}$ maser line, and (3) the $\mathrm{CH}_{3} \mathrm{CN}\left(2_{k}-1_{k}\right)$ transitions, the resolution is doubled to better resolve the line structure.

The phase calibrator (J1744-3116) was observed approximately every 15 minutes. The bandpass calibrator was J1733-1304. The data were flux calibrated using 3C286, observed at elevations comparable to the Galactic center sources $\left(\sim 15^{\circ}-30^{\circ}\right)$. 


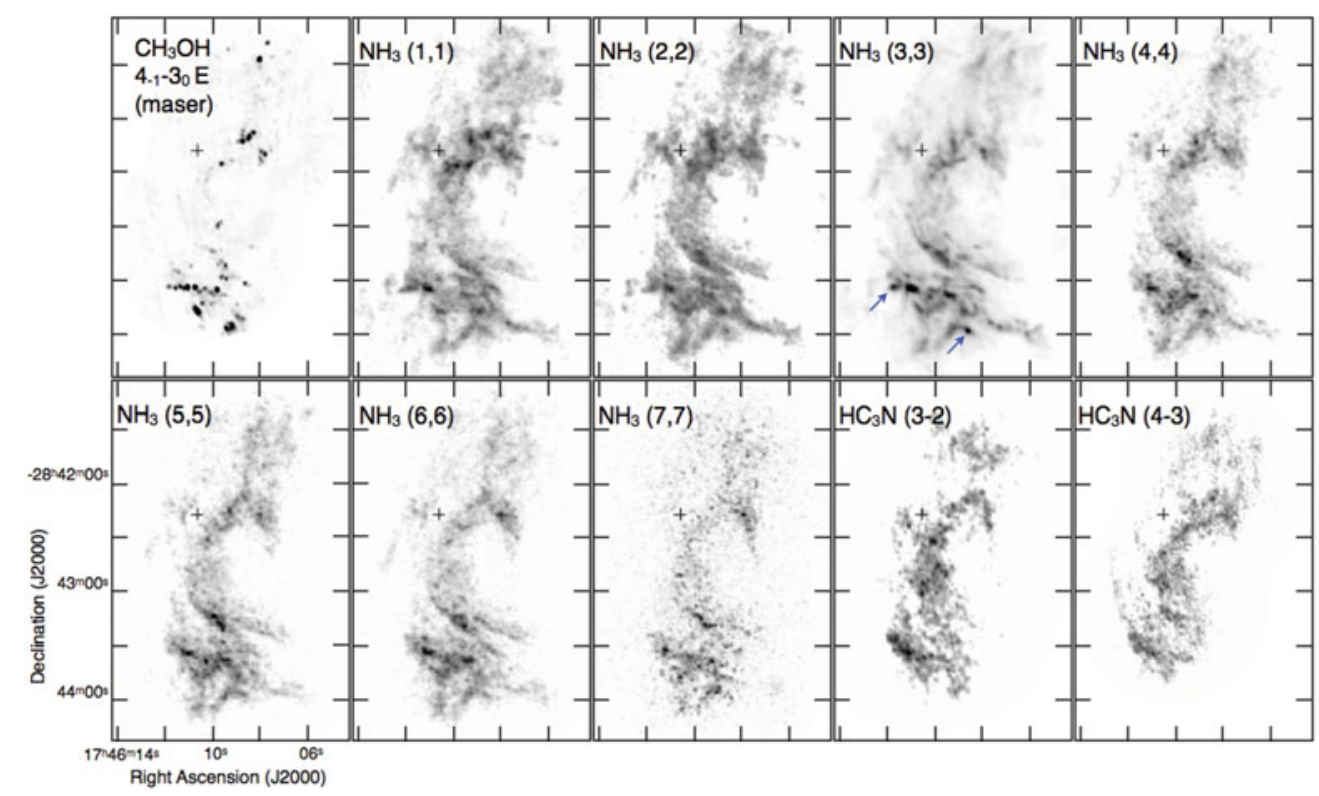

Figure 1. Peak intensity maps of molecular lines in M0.25 +0.01, including $36 \mathrm{GHz}$ methanol masers, 7 transitions of $\mathrm{NH}_{3}$, and two transitions of $\mathrm{HC}_{3} \mathrm{~N}$. The cross indicates the location of an $\mathrm{H}_{2} \mathrm{O}$ maser (Lis et al. 1994) coinciding with a dust continuum peak (Kauffmann et al. 2013). Examples of candidate $\mathrm{NH}_{3}(3,3)$ masers are indicated with arrows.

After calibration with the $C A S A$ reduction package, the data were imaged using the CLEAN algorithm in $C A S A$ and a CLEAN cycle of several thousand iterations, resulting in noise in the final images which was typically $\sim 1 \mathrm{mJy}^{\text {beam }}{ }^{-1}$ channel $^{-1}$ for the spectral line images. The full width at half maximum (FWHM) of the synthesized clean beam ranged from 2 ". $3 \times 2$ ". 5 at $25 \mathrm{GHz}$ to $11^{\prime \prime} 9 \times 22^{\prime \prime} .2$ at $27 \mathrm{GHz}$ and $1^{\prime \prime} .5 \times 1{ }^{\prime \prime} .7$ at $36 \mathrm{GHz}$.

\section{Results}

We present preliminary images of M0.25+0.01, clouds near Sgr A, and Sgr B2.

M0.25 + 0.01: This cloud is one of the most massive in the central molecular zone (CMZ) $\left(\mathrm{M} \sim 1-2 \times 10^{5} \mathrm{M}_{\odot}\right.$; Lis et al. 1994, Longmore et al. 2012). However, unlike other massive CMZ clouds, there is no evidence in this cloud for ongoing star formation apart from a single water maser (Lis et al. 1994, Longmore et al. 2012, Kauffmann et al. 2013).

Mapping this cloud, we find the morphology of the $\mathrm{NH}_{3}$ transitions is almost identical (apart from a few candidate masers in the $(3,3)$ lines; Figure 1): there is no sign of temperature gradients. The $(1,1)$ and $(2,2)$ transitions are both very optically-thick, $(\tau$ up to 4-7), as measured from the ratio of their hyperfine satellites. From the optically-thin $(4,4),(5,5)$, and $(7,7)$ lines, we measure a median temperature of $\sim 100 \mathrm{~K}$.

The distribution of $\mathrm{HC}_{3} \mathrm{~N}$ is similar to $\mathrm{NH}_{3}$, but is stronger toward the center of the cloud, and may trace denser gas. We also detect numerous $36.1 \mathrm{GHz} \mathrm{CH}_{3} \mathrm{OH}$ point sources, the distribution of which closely follows the morphology of the $\mathrm{NH}_{3}$ gas (Figure 1). The majority of the $\mathrm{CH}_{3} \mathrm{OH}$ sources are relatively weak, with intensities of 0.5 - $1 \mathrm{Jy}_{\text {beam }}{ }^{-1}$. However, more than $80 \%$ can be identified as masers, having brightness temperatures greater than the hottest gas identified in this cloud $(400 \mathrm{~K}$; Mills \& Morris 2013). Dozens of such candidate masers are found in every cloud surveyed. As 


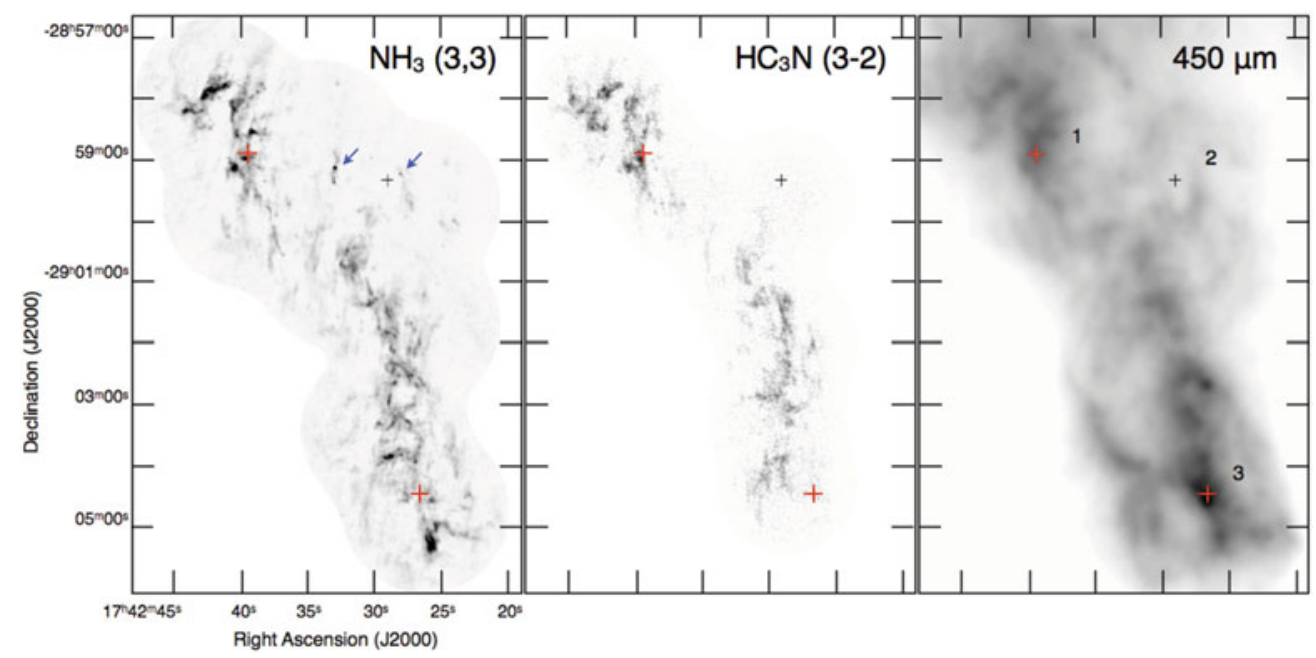

Figure 2. Peak intensity maps of $\mathrm{NH}_{3}(3,3)$ (left) and $\mathrm{HC}_{3} \mathrm{~N} 3-2$ (center) in Sgr A, compared to 450 micron dust continuum (Right; Pierce-Price et al. 2000). Crosses indicate dust continuum peaks in $\mathrm{M}-0.02-0.07$ (1), $\mathrm{M}-0.13-0.08$ (3), and the location of the supermassive black hole Sgr A* (2). Examples of candidate $\mathrm{NH}_{3}(3,3)$ masers are indicated with arrows. [A COLOR VERsion is AVAiLABLe online.]

these are Class I masers, they are indicative of shocks, but in the turbulent environment of the Galactic center, they likely do not indicate star formation.

Sgr A: There are three main clouds near Sgr A: M-0.02-0.07, M-0.13-0.08, and the circumnuclear disk (CND), which surrounds the supermassive black hole, Sgr A*. In projection, these clouds lie within the central $10 \mathrm{pc}$ of the Galaxy, and are believed to be physically close as well, based upon their interactions with each other and with the gravitational potential of Sgr A* (Coil \& Ho 2000, Herrnstein \& Ho 2005).

The strongest sources of $\mathrm{NH}_{3}$ emission are $\mathrm{M}-0.02-0.07$, and a clump at the southern tip of $\mathrm{M}-0.13-0.08$ (Figure 2). However, this clump is entirely absent in $\mathrm{HC}_{3} \mathrm{~N}$. As in $\mathrm{M} 0.25+0.01$, there is also no $\mathrm{NH}_{3}$ or $\mathrm{HC}_{3} \mathrm{~N}$ detected toward the strongest dust continuum peak in $\mathrm{M}-0.13-0.08$ and its associated $\mathrm{H}_{2} \mathrm{O}$ masers (Guesten \& Downes 1983; Sjouwerman et al. 2002).

$\mathrm{HC}_{3} \mathrm{~N}$ is not detected toward the $\mathrm{CND}$, which is either an indication that gas in this region is less dense, or that $\mathrm{HC}_{3} \mathrm{~N}$ is under-abundant or destroyed in this environment (Martín et al. 2012). $\mathrm{NH}_{3}$ emission from the CND is weak in comparison to emission from $\mathrm{M}-0.02-0.07$ and $\mathrm{M}-0.13-0.08$, although several strong, compact sources of $\mathrm{NH}_{3}(3,3)$ emission (likely masers) are found here, coincident with observed 36 and $44 \mathrm{GHz} \mathrm{CH}_{3} \mathrm{OH}$ masers (Sjouwerman et al. 2010, Yusef-Zadeh et al. 2008).

Sgr B2: This is the most massive Galactic center cloud, and hosts extremely active star formation traced by dozens of ultracompact HiI regions (e.g., De Pree et al. 1998).

The structure of the cloud, as seen in $\mathrm{NH}_{3}(3,3)$, is a wide filament oriented in the southeast/northwest direction (Figure 3). $\mathrm{NH}_{3}(3,3)$ emission is detected from the ' $\mathrm{N}$ ' and 'M' sub-millimeter cores, as well as likely $(3,3)$ masers in the south (Martín-Pintado et al. 1999). Emission from most other molecules is confined to $\mathrm{N}$, although all three submillimeter cores $(\mathrm{N}, \mathrm{M}, \mathrm{S})$ are detected in $\mathrm{J}_{2}-\mathrm{J}_{1}$ E-type $\mathrm{CH}_{3} \mathrm{OH}$ transitions. In the primary hot core in $\mathrm{N}$ (SMA-1, v $63 \mathrm{~km} \mathrm{~s}^{-1}$ ), emission from these lines of $\mathrm{CH}_{3} \mathrm{OH}$ and its ${ }^{13} \mathrm{C}$ isotopologue, as well as from a torsionally-excited line of $\mathrm{A}^{+}$-type $\mathrm{CH}_{3} \mathrm{OH}\left(12_{2}-11_{1}\right.$, $\mathrm{v}_{T}=1$ ) traces a ringlike structure of diameter $5^{\prime \prime}$ (Figure 3), first mapped in $\mathrm{CH}_{3} \mathrm{CH}_{2} \mathrm{CN}$ 


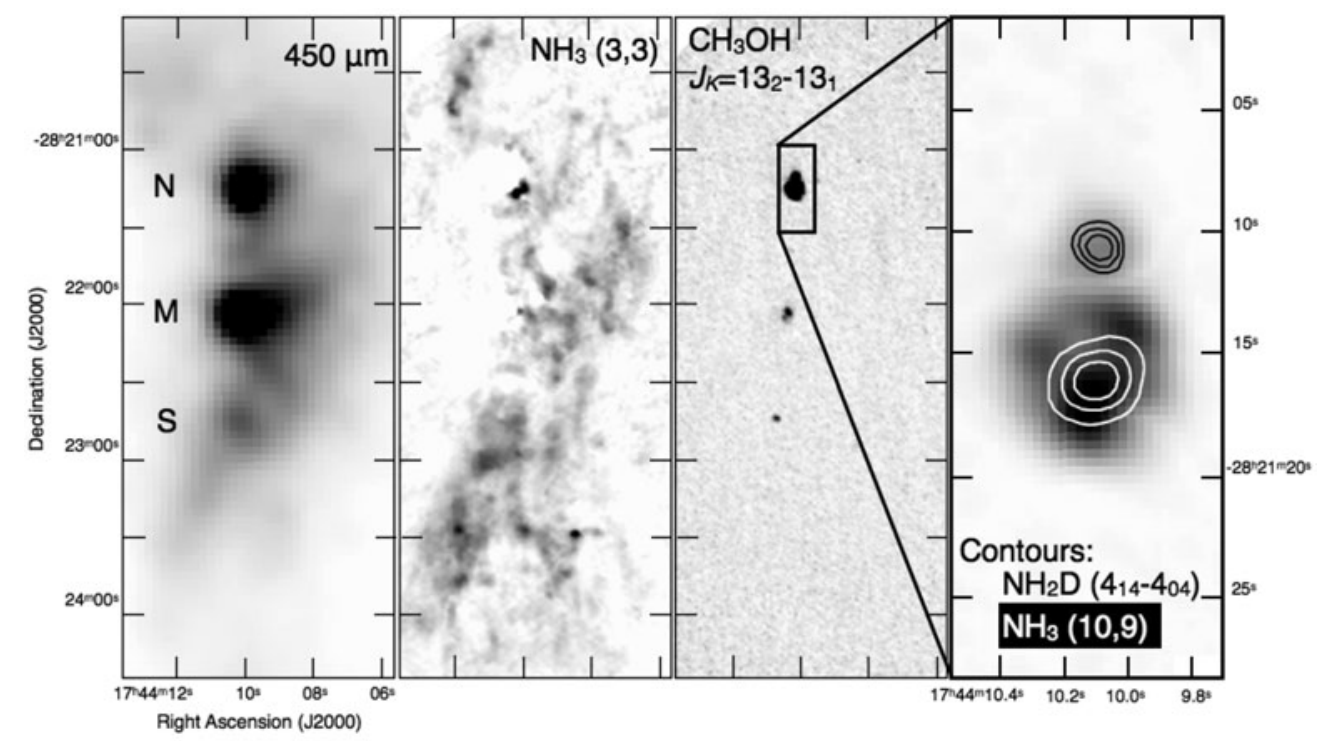

Figure 3. Peak intensity maps of $\mathrm{NH}_{3}(3,3)$ (center) and $\mathrm{CH}_{3} \mathrm{OH} \quad 13_{2}-13_{1}$ (Rightmost two panels) in Sgr B2, compared to 450 micron dust continuum (Left; Pierce-Price et al. 2000). The far-right panel shows a zoom of Sgr B2-N and the two hot cores: SMA-1 (white contours) the most chemically-rich source in this survey and the location of the strongest non-metastable $\mathrm{NH}_{3}$ emission, and SMA-2 (black contours), where we detect $\mathrm{NH}_{2} \mathrm{D}$.

(Hollis et al. 2003). A second hot core in $\mathrm{N}$ (SMA-2, $v \sim 75 \mathrm{~km} \mathrm{~s}^{-1}, 5^{\prime \prime}$ to the north; Liu \& Snyder 1999, Qin et al. 2011) is also seen in the $\mathrm{J}_{2}-\mathrm{J}_{1}$ lines of $\mathrm{CH}_{3} \mathrm{OH}$ and ${ }^{13} \mathrm{CH}_{3} \mathrm{OH}$.

Metastable $\mathrm{NH}_{3}$ emission in SMA-1, as previously detected by Vogel et al. (1987), is extremely optically thick: hyperfine satellites are clearly detected in all lines, including $(9,9)$. Emission from the $\mathrm{NH}_{3}$ satellite lines (which are more optically thin than the main lines) as well as the optically-thin isotopologue ${ }^{15} \mathrm{NH}_{3}$, peaks on the southern edge of this ring, and is roughly co-spatial with emission from vibrationally-excited transitions of $\mathrm{HC}_{3} \mathrm{~N}$ and non-metastable $\mathrm{NH}_{3}$. However, the $4_{14}-4_{04}$ transition of singly-deuterated ammonia $\left(\mathrm{NH}_{2} \mathrm{D}\right)$ is not detected toward SMA-1, and is only seen toward SMA-2, suggesting the deuterated fraction of this core may be higher than previously estimated for 'N' as a whole (Peng et al. 1993).

\section{References}

Bally, J., Stark, A., Wilson, T. L., \& Henkel, C. 1987, ApJS 65, 13

Coil, A. L. \& Ho, P. T. P. 2000, ApJ 533, 245

De Pree, C. G., Goss, W. M., \& Gaume, R. A. 1998, ApJ 500, 847

Güsten, R. \& Downes, D. 1983, A\&A 117, 343

Herrnstein, R. M. \& Ho, P. T. P. 2005, ApJ 620, 287

Hollis, J. M., Pedelty, J. A., Boboltz, D. A., Liu, S.-Y., Snyder, L. E., et al. 2005, ApJ 596, L235

Jones, P. A., Burton, M. G., Cunningham, M. R., Tothill, N., \& Walsh, A. 2005, MNRAS 433, 221

Kauffmann, J., Pillai, T., \& Zhang, Q. 2013, ApJ 765, L35

Lis, D. C., Menten, K. M., Serabyn, E., \& Zylka, R. 1994, ApJ 423, L39

Liu, S.-Y. \& Snyder, L. E. 1999, ApJ 523, 683

Longmore, S. N., Rathborne, J., Bastian, N., Alves, J., Ascenso, J., et al. 2005, ApJ 746, 117

Martín, S., Martín-Pintado, J., Montero-Castaño, M., Ho, P. T. P., et al. 2012, A $\varepsilon A$ 539, 21 
Martín-Pintado, J., Gaume, R. A., Rodríguez-Fernández, N., et al. 1999, ApJ 519, 667

Mills, E. A. C. \& Morris, M. R. 2013, ApJ 772, 105

Peng, Y., Vogel, S. N., \& Carlstrom, J. E. 1993, ApJ 418, 255

Pierce-Price, D., Richer, J. S., Greaves, J. S., Holland, W. S., et al. 2000, ApJ 545, L121

Qin, S. L., Schilke, P., Rolffs, R., Comito, C., Lis, D. C., \& Zhang, Q. 2011, A\&A A 530, L9

Sjouwerman, L. O., Lindqvist, M., van Langevelde, H. J., \& Diamond, P. J. 2002, A\&A 391, 967

Sjouwerman, L. O., Pihlström, Y. M., \& Fish, V. L. 2010, ApJ 710, L111

Vogel, S. N., Genzel, R., \& Palmer, P. 1987, ApJ 316, 243

Yusef-Zadeh, F., Braatz, J., Wardle, M., \& Roberts, D. 2008, ApJ 683, L147 